\title{
Possible role of magnetosphere-ionosphere coupling in auroral arc generation
}

\author{
W. Lyatsky ${ }^{1}$, A. M. Hamza \\ Physics Department, University of New Brunswick, N.B., Canada \\ ${ }^{1}$ Polar Geophysical Institute, Apatity, Russia \\ Received: 17 December 1999 / Revised: 29 March 2000 / Accepted: 3 May 2000
}

\begin{abstract}
Three models for the magnetosphere-ionosphere coupling feedback instability are considered. The first model is based on demagnetization of hot ions in the plasma sheet. The instability takes place in the global magnetosphere-ionosphere system when magnetospheric electrons drift through a spatial gradient of hot magnetospheric ion population. Such a situation exists on the inner and outer edges of the plasma sheet where relatively cold magnetospheric electrons move earthward through a radial gradient of hot ions. This leads to the formation of field-aligned currents. The effect of upward field-aligned current on particle precipitation and the magnitude of ionospheric conductivity leads to the instability of this earthward convection and to its division into convection streams oriented at some angle with respect to the initial convection direction. The growth rate of the instability is maximum for structures with sizes less than the ion Larmor radius in the equatorial plane. This may lead to formation of auroral arcs with widths about $10 \mathrm{~km}$. This instability explains many features of such arcs, including their conjugacy in opposite hemispheres. However, it cannot explain the very high growth rates of some auroral arcs and very narrow arcs. For such arcs another type of instability must be considered. In the other two models the instability arises because of the generation of Alfven waves from growing arc-like structures in the ionospheric conductivity. One model is based on the modulation of precipitating electrons by field-aligned currents of the upward moving Alfven wave. The other model takes into consideration the reflection of Alfven waves from a maximum in the Alfven velocity at an altitude of about $3000 \mathrm{~km}$. The growth of structures in both models takes place when the ionization function associated with upward field-aligned current is shifted from the edges of enhanced conductivity structures toward their centers. Such a shift arises because the structures move at a velocity different from the $\mathbf{E} \times \mathbf{B}$
\end{abstract}

Correspondence to: W. Lyatsky

e-mail: lyatsky@unb.ca drift. Although both models may work, the growth rate for the model, based on the modulation of the precipitating accelerated electrons, is significantly larger than that of the model based on the Alfven wave reflection. This mechanism is suitable for generation of auroral arcs with widths of about $1 \mathrm{~km}$ and less. The growth rate of the instability can be as large as $1 \mathrm{~s}^{-1}$, and this mechanism enables us to justify the development of auroral arcs only in one ionosphere. It is hardly suitable for excitation of wide and conjugate auroral arcs, but it may be responsible for the formation of small-scale structures inside a wide arc.

Key words: Ionosphere (auroral ionosphere) Magnetospheric physics (auroral phenomena; magnetosphere-ionosphere interactions)

\section{Introduction}

It was shown by many scientists that the magnetospheric convection may be unstable and divided into convection streams (e.g., Sato and Holzer, 1973; Sato, 1978; Leontyev and Lyatsky, 1982; Trakhtengertz and Feldstein, 1984; Lyatsky, 1987; Watanabe and Sato, 1988; Lysak, 1991; Kozlovsky and Lyatsky, 1994, 1999). Sato and Holzer (1973) were the first to propose a selfconsistent model for the magnetospheric convection stratification. They showed that field-aligned currents of an Alfven wave emitted from an ionospheric inhomogeneity, while reflected from the conjugate ionosphere, may lead to an additional increase in ionization inside growing structures and to the development of feedback instability. Later other mechanisms for convection stratification were proposed: the interchange or shear flow instability (e.g., Roux et al., 1991; Samson et al., 1996), a magnetosphere-ionosphere coupling feedback instability in the lower magnetosphere (e.g., Leontyev and Lyatsky, 1982; Trakhtengertz and Feldstein, 1984; 
Lysak, 1991), and a magnetosphere-ionosphere coupling instability including an active role of hot magnetospheric plasma (Lyatsky, 1987; Kozlovsky and Lyatsky, 1994, 1999). All these authors suggested that the convection streams, appearing as a result of this instability, might be responsible for auroral arc generation. An important role of magnetosphere-ionosphere coupling in auroral arc generation was recently demonstrated by Newell et al. (1996a, b) who showed a strong suppression of auroras in the sunlit ionosphere.

The physics of the magnetosphere-ionosphere coupling (MIC) feedback instability can be explained as follows. A band of enhanced ionospheric conductivity immersed in an external ionospheric electric field produces a polarization electric field that propagates into the magnetosphere. Field-aligned currents, associated with this polarization electric field, provide no instability, because they are formed at the edges of the band. Therefore, they only cause motion of the band toward upward field-aligned current, which causes an additional ionization. While propagating into the magnetosphere, the polarization electric field, through the redistribution of magnetospheric currents over the inhomogeneity region or through the reflection of the Alfven wave associated with these initial field-aligned currents, can provide secondary field-aligned currents. The instability takes place when the secondary upward field-aligned currents appear inside the band of enhanced conductivity. This leads to the growth of the inhomogeneity.

Three different ways to provide MIC feedback instability have been proposed:

1. Through demagnetization of hot ions in the plasma sheet and the generation of Hall magnetospheric currents in the vicinity of the inner edge of the plasma sheet where a forbidden region for warm ions exists (Lyatsky, 1987; Kozlovsky and Lyatsky, 1994, 1999).

2. Through the reflection of an Alfven wave emitted by an ionospheric inhomogeneity, from some region in the magnetosphere, as suggested by Sato and Holzer (1973), Sato (1978), Trakhtengertz and Feldstein (1984), Watanabe and Sato (1988) and Lysak (1991).

3 . Through modulation of particle precipitation by fieldaligned currents of this Alfven wave as suggested by Leontyev and Lyatsky (1982).

There are also two different regions where the instability may be triggered. It may take place in the global magnetosphere-ionosphere system, as considered by Sato and Holzer (1973) and Kozlovsky and Lyatsky (1994, 1999). It can also arise in the lower magnetosphere between the ionosphere and the electron acceleration region at about $1 R_{E}$ (Leontyev and Lyatsky, 1982), or between the ionosphere and the region of maximum Alfven velocity at an altitude of about $3000 \mathrm{~km}$ (e.g., Trakhtengertz and Feldstein, 1984; Lysak, 1991).

Our aim is to present some recent results related to the study of these types of magnetosphere-ionosphere coupling feedback instabilities and discuss their possible application to auroral are generation.

\section{Three models for magnetosphere-ionosphere coupling feedback instability}

In this section we consider the three models for magnetosphere-ionosphere coupling feedback instability cited. (1) the ion demagnetization model by Lyatsky (1987) and Kozlovsky and Lyatsky (1994, 1999); (2) the Alfven wave oscillation model by Sato and Holzer (1973), Sato (1978), Trakhtengertz and Feldstein (1984), Watanabe and Sato (1988), and Lysak (1991); and (3) the particle modulation model by Leontyev and Lyatsky (1982). In Sect. 2.1 we examine the ion demagnetization model, in Sects. 2.2 and 2.3 we discuss two models for the excitation of the instability in the lower magnetosphere, as based on the modulation of precipitating electrons by field-aligned currents of the Alfven wave and on the reflection of the wave from a maximum of the Alfven velocity at about $3000 \mathrm{~km}$, respectively.

\subsection{Ion demagnetization model for magnetosphere- ionosphere coupling feedback instability in the global magnetosphere-ionosphere system}

This model includes the effect of ion demagnetization in the magnetotail plasma sheet that leads to the formation of magnetospheric currents closing field-aligned currents. A shift of upward field-aligned currents toward the center of a conductive band in this model is provided by the generation in the plasma sheet of a secondary field-aligned current $j_{z}=\operatorname{div} \mathbf{J}_{M}$, where $\mathbf{J}_{M}$ are transverse magnetospheric currents. This model is suitable for generating relatively wide arcs (10 km and more) when the spatial shift of the Alfven wave can be neglected, which allows us to use a quasi-stationary approach.

This instability was studied earlier by Lyatsky (1987) and Kozlovsky and Lyatsky $(1994,1999)$ in some specific cases. We will examine this instability in a more precise study. As opposed to the paper by Kozlovsky and Lyatsky (1999), where the cause for ion demagnetization in the plasma sheet is considered to be the finite ion Larmor radius in the plasma sheet, we will now study the instability in the vicinity of the inner edge of the plasma sheet, where hot plasma sheet ions are partially demagnetized because of their azimuthal motion around the Earth and the formation of a forbidden region for hot ions. We then discuss the effect of the finite ion Larmor radius in the magnetotail plasma sheet.

For simplicity we assume that the plasma sheet consists of hot (about $10 \mathrm{keV}$ ) ions and relatively cold electrons. The instability takes place when magnetospheric electrons drift through a spatial gradient of hot magnetospheric ion content. Such a situation occurs around the inner edge of the plasma sheet where relatively cold magnetospheric electrons drift earthward through a radial gradient of hot ions. Because the number of ions in drifting magnetic tubes decrease, some of the superfluous electrons must leave these tubes for the ionosphere providing the formation of upward 


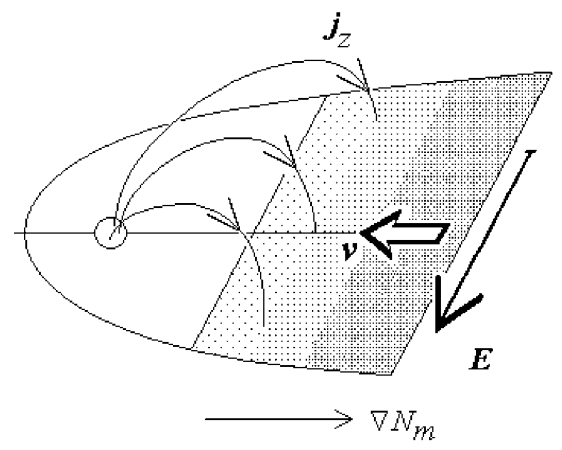

Fig. 1. Formation of field-aligned currents around the inner edge of the plasma sheet. The earthward $\mathbf{E} \times \mathbf{B}$ drift of electrons through the given hot ion distribution leads to their precipitation into the ionosphere, which yields upward field-aligned currents. $E$ is the external electric field, $v$ is the electron $\mathbf{E} \times \mathbf{B}$ drift velocity, $j_{z}$ are fieldaligned currents, $N_{m}$ represents the content of hot ions in a magnetic tube of unit cross section on the ionospheric level, and $\nabla N_{m}$ is directed tailward (poleward at the ionospheric level)

field-aligned currents as shown in Fig. 1. We note that although from Fig. 1 one may think that upward fieldaligned currents should be formed in all nightside magnetosphere, in fact these currents are turned toward the morning meridian. The cause of this turning is considered, for instance, by Jaggi and Wolf (1973), Lyatsky and Maltsev (1983), Volkov et al. (1985), and Lyatsky and Hamza (2000).

This mechanism for the development of field-aligned currents on the plasma sheet inner boundary is traditionally believed to be the main cause of formation of region-2 field-aligned currents on the night side (e.g., Jaggi and Wolf, 1973; Lyatsky and Maltsev, 1983; Volkov et al., 1985; Potemra, 1994). However, this situation is unstable and leads to breaking up the convection into convection streams that may be associated with auroral arcs.

The physics of this instability is explained in Figs. 2 and 3. Let us assume that a narrow band of enhanced conductivity appears in the ionosphere. The ionospheric electric field leads to the polarization of this band and to the appearance of electric charges on its edges. For some orientation of the band, such as shown in Figs. 2 and 3, this polarization electric field, while propagating into the magnetosphere, provides an increase in the earthward convection velocity inside the band. This in turn leads to an increase in electron precipitation and to the growth of ionospheric conductivity in the band. Figure 3 shows the formation of the polarization electric field leading to the instability development at the ionospheric level.

Let us assume the warm ion distribution in the magnetotail plasma sheet to be given and constant. We also assume that at some distance from the Earth there exists an inner boundary of the plasma sheet caused by the formation of a forbidden region for warm ions, where their azimuthal drift in the curved geomagnetic field becomes comparable to the $\mathbf{E} \times \mathbf{B}$ drift. As a result, hot magnetospheric ions cannot penetrate into the inner magnetosphere (e.g., Jaggi and Wolf, 1973; Lyatsky and Maltsev, 1983; Wolf, 1995). Experimental results are

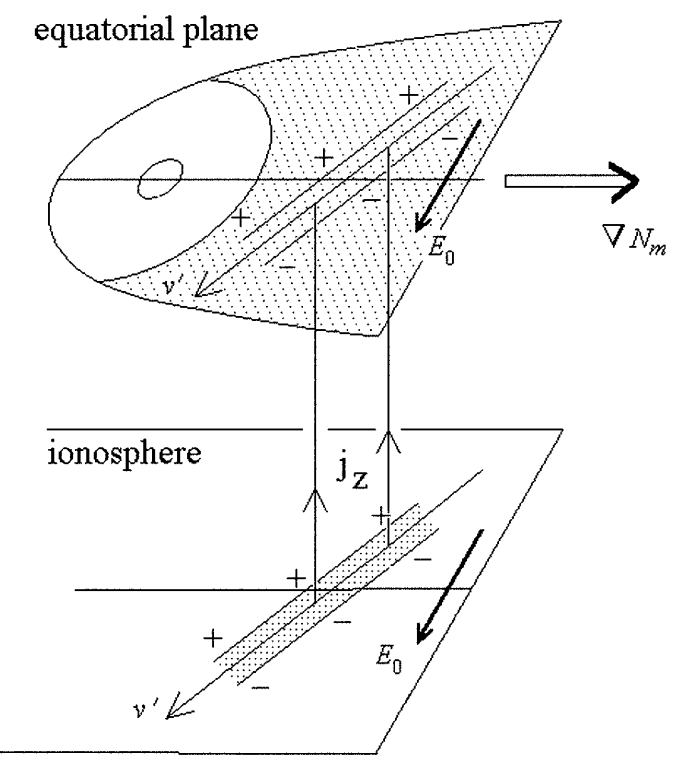

Fig. 2. A scheme explaining the instability development. Polarization charges arising at the edges of bands of enhanced ionospheric conductivity mapped into the equatorial plane lead to an increase of the earthward convection flow inside the bands. This produces an additional increase in upward field-aligned current inside the bands, which leads to the instability development. $E$ is the external electric field, $v^{\prime}$ is the increment in the convection velocity inside the bands, $j_{z}$ is the upward field-aligned current. The gradient of hot magnetospheric ions $\nabla N_{m}$ is also shown

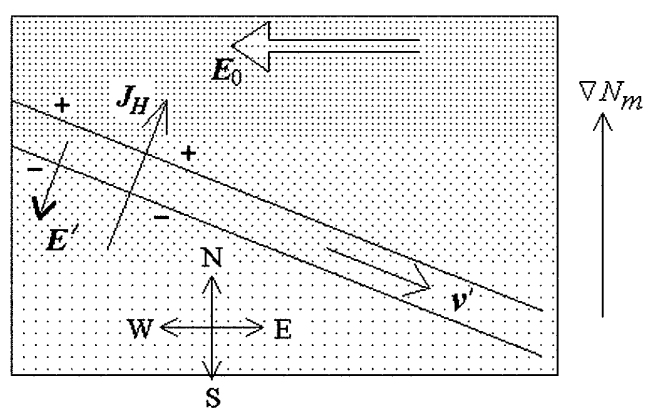

Fig. 3. A scheme explaining the instability development at the ionospheric level. A polarization electric field $E^{\prime}$ arising inside the band produces an increase of the $\mathbf{E} \times \mathbf{B}$ drift of magnetospheric electrons along the band. In the presence of a radial gradient of the electron content in the plasma sheet, this leads to an increase in upward field-aligned currents inside the band and to the instability development. $\mathbf{E}_{0}$ is the external electric field, $\mathbf{J}_{H}$ is the component of the Hall ionospheric current across the band, $\boldsymbol{v}^{\prime}$ is the increment in the convection velocity. The distribution of hot magnetospheric ions $N_{m}$ is also shown by dotted areas as projected onto the ionosphere

supporting this theoretical prediction (Wolf, 1995). The existence of the forbidden region implies that the warm ion content in a given magnetic field tube decreases earthward. We also assume that there is an earthward drift of cold electrons through the given distribution of ions.

Assuming magnetic field lines to be quasi-equipotential and integrating the magnetospheric equation along the magnetic field line, we can reduce the threedimensional set of equations to a two-dimensional set at 
the ionospheric level (Kozlovsky and Lyatsky, 1994, 1999).

We use the continuity equation for ionospheric currents

$j_{z}=\operatorname{div}_{\perp} \mathbf{J}=\nabla_{\perp} \cdot\left(\Sigma_{P} E+\Sigma_{H} b \times E\right)$

where $j_{z}$ is the field-aligned current (positive direction is along the magnetic field); $\mathbf{J}$ is the height-integrated ionospheric current; $\Sigma_{P}$ and $\Sigma_{H}$ are the height-integrated Pedersen and Hall ionospheric conductivities, respectively; $\mathbf{E}$ is the electric field; and $\mathbf{b}$ is the unit vector along the magnetic field.

We also use the continuity equation for magnetospheric electrons, which may be written at the ionospheric level in following form:

$\frac{\partial N_{m}}{\partial t}+\nabla_{\perp} \cdot\left(N_{m} v\right)=(1 / e) j_{z}$

where $N_{m}$ is the number of electrons in a magnetic field tube of unit cross-section at the ionospheric level (which is equal to the content of hot ions) extending from the equatorial plane to the ionosphere. The distribution of $N_{m}$ is assumed to be given, and $\boldsymbol{v}$ is the $\mathbf{E} \times \mathbf{B}$ drift velocity of cold electrons.

The ionospheric plasma density is derived from the ionization balance equation, which can be written in the following form:

$\frac{\partial n}{\partial t}+\nabla_{\perp} \cdot(n v)=\chi \frac{j_{z 0}}{e \Delta z}\left|\frac{j_{z}}{j_{z 0}}\right|^{\mu}-\alpha n^{2}$

where $n$ is the average value of the plasma density in the $\mathrm{E}$ region in the height interval $\Delta z, e$ is the electron charge, and $\alpha$ is the recombination factor for the ionospheric $\mathrm{E}$ region. The factor $\chi=1+\delta \varepsilon / \varepsilon_{i}$ shows the efficiency of ionization produced by accelerated precipitating electrons; here $\varepsilon$ is the energy of precipitating electrons in $\mathrm{eV}, \varepsilon_{i}$ is the ionization potential $(\sim 16 \mathrm{eV})$, and the factor $\delta \approx 0.5$. The value $j_{z 0}$ means the undisturbed field-aligned current, and the exponent $\mu$ on the right hand side of Eq. (3) is thought to be about 3 for the case when field-aligned current $j_{z}$ is associated with accelerated electrons, and about 1 for the case when $j_{z}$ is carried by cold electrons (e.g., Lyatsky, 1999).

We apply these equations to the case of the inner boundary of the plasma sheet. Assuming $\partial N_{m} / \partial t=0$, in the linear approximation for a plane wave $\exp [-i \omega t+$ $i(\mathbf{k} \cdot \mathbf{r})]$, and assuming $\Sigma_{P}^{\prime} / \Sigma_{P 0}=n^{\prime} / n_{0}$, where $n^{\prime}$ and $\Sigma_{P}^{\prime}$ represent perturbations, yields the following dispersion equation:

$\omega=\mathbf{k} \cdot \mathbf{v}_{0}-\alpha n_{0} \mu \frac{A+C}{1+C^{2}}-i \alpha n_{0}\left(\mu \frac{A C-1}{1+C^{2}}+2\right)$

where

$A=\frac{1}{j_{z 0}} \mathbf{k} \cdot\left(\mathbf{J}_{P 0}+\xi \mathbf{J}_{H 0}\right)$

$C=\frac{k^{2}}{k_{e}} \frac{\Sigma_{P 0} E_{e 0}}{j_{z 0}}$

where $k_{e}$ is the eastward component of the wave vector, and $E_{e}$ is the eastward component of the electric field, and $\xi=\Sigma_{H}^{\prime} / \Sigma_{P}^{\prime}$. The subscript " 0 " means "undisturbed" values. The instability takes place when

$\mu \frac{A C-1}{1+C^{2}}+2<0$

That implies $A C<0$ or $k_{e} E_{e 0}\left(\mathbf{k} \cdot \mathbf{J}_{e f}\right)<0$, where $\mathbf{J}_{e f}=\mathbf{J}_{P 0}+\xi \mathbf{J}_{H 0}$. Since $E_{e}$ is directed westward, $E_{e}$ is negative. Then we obtain the following condition for the instability

$k_{e}\left(\mathbf{k} \cdot \mathbf{J}_{e f}\right)>0$

If the Hall to Pedersen conductivity ratio $\xi \approx 1$, the effective current $\mathbf{J}_{e f}=\mathbf{J}_{P 0}+\xi \mathbf{J}_{H 0}$ is the ionospheric current, but if $\xi>1$, the current $\mathbf{J}_{\text {ef }}$ tends to approach the Hall current.

The expected orientation of unstable wave vectors for a case $\xi \gg 1$ and $\mathbf{J}_{e f}$ directed approximately along $\mathbf{J}_{H 0}$ is shown in Fig. 4. The range of possible orientations for unstable wave vectors, as determined by Eq. (7), is a narrow region around the angle of $(\phi / 2)$, where the growth rate is maximum. Assuming the characteristic distance $\Delta x$ for variation of $E_{x 0}$ to be much larger than the wave length $\lambda=2 \pi / k$, we obtain $|C| \gg 1$. We then obtain the following expression for the growth rate:

$\operatorname{Im} \omega=-\alpha n_{0}\left(\mu \frac{k_{e}}{k^{2}} \frac{\mathbf{k} \cdot \mathbf{J}_{e f}}{\mathbf{J}_{P e 0}}+2\right)$

where $\mathbf{J}_{P e 0}$ is the eastward component of the Pedersen current, $\mu$ is a factor close to 3 , and $\xi=\Sigma_{H} / \Sigma_{P}$. Assuming again $\mathbf{J}_{e f} \approx \xi \mathbf{J}_{H_{0}}$ and $k_{e} / k=\sin (\phi / 2)$, we obtain the following condition for the instability

$\mu \xi \tan (\phi / 2)>4$

Near midnight for $\mu=3$ and $\xi=3$, the instability takes place for $\phi>45^{\circ}$ where $\phi$ is the angle between $\mathbf{k}$ and the eastward direction. The characteristic time of instability growth may be as small as about 1-2 min but it cannot be less than the Alfven wave bounce period. Unstable orientations are shown in Fig. 5.

For the real part of the complex frequency we obtain

$\operatorname{Re} \omega=\mathbf{k} \cdot \mathbf{v}_{0}-\mu \alpha n_{0} \frac{A+C}{C^{2}}$

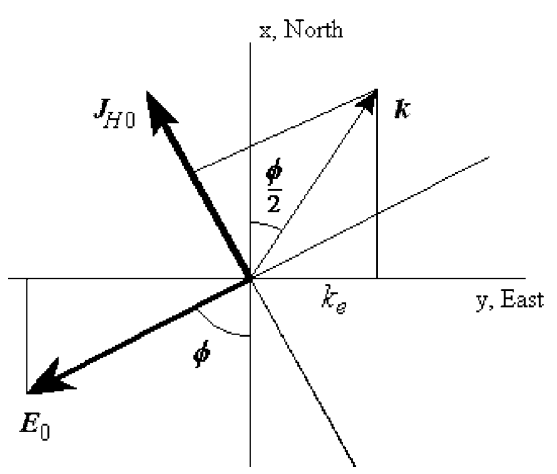

Fig. 4. The expected orientation of wave vector for growing structures. $\mathbf{E}_{0}$ is the external electric field, $\mathbf{J}_{H}$ is the Hall current, $\mathbf{k}$ is the wave vector. We note that for a given orientation of $\mathbf{E}_{0}$, typical for the midnight ionosphere, $\mathbf{k}$ is directed poleward 


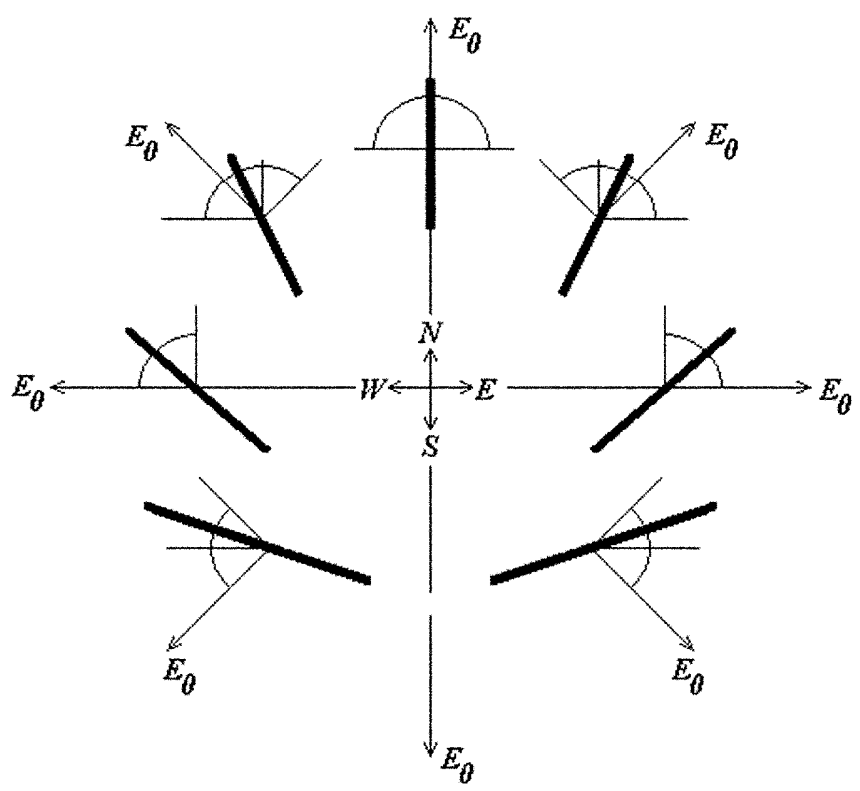

Fig. 5. Expected orientations of growing convection streams (as shown by bold lines) for different directions of the external electric field $\mathbf{E}_{0}$. The range of permitted orientations with a positive growth rate is also shown

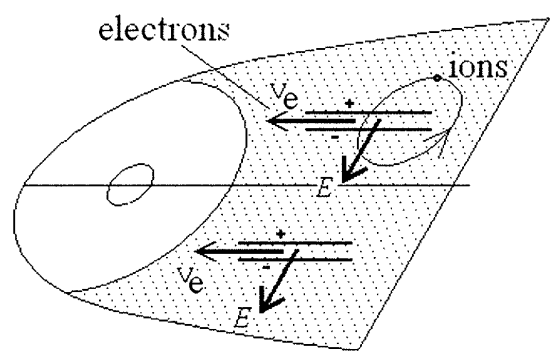

Fig. 6. A scheme explaining the formation of a Hall current in the equatorial plane inside narrow structures with size much less than the ion Larmor radius. The $\mathbf{E} \times \mathbf{B}$ drift of electrons along the structures leads to the Hall magnetospheric current directed along these structures. Ions, because of their large Larmor radius, do not participate in this motion. $\mathbf{E}$ is the polarization electric field inside these structures, $v_{e}$ is the electron $\mathbf{E} \times \mathbf{B}$ drift velocity along the structures

In agreement with Kozlovsky and Lyatsky (1999), because the factor $C$ takes a large value, the magnitude of $\operatorname{Re} \omega$ in the linear approximation is much less than that of the growth rate.

For small-scale structures with sizes less than the ion Larmor radius in the equatorial plane, electrons can drift along such structures, whereas ions do not participate in this motion (Fig. 6). This leads to drifting electrons through an immobile ion population, which in turn leads to the development of Hall magnetospheric currents inside the structures. The presence of a radial gradient of hot ions leads to the generation of upward field-aligned currents similar to the previous case. The growth rate of the instability is derived using the same expression as for the previous case but multiplied by a coefficient such that the growth rate increases with the magnitude of the wave vector (Kozlovsky and Lyatsky, 1999).

Thus, this instability takes place when magnetospheric electrons drift through the spatial gradient of a hot magnetospheric ion population. Such a situation occurs on the inner and outer edges of the plasma sheet where relatively cold magnetospheric electrons move earthward through a radial gradient of hot ions. This leads to the motion of electrons along magnetic field lines and to the formation of field-aligned currents. The effect of upward field-aligned currents on particle precipitation and on the magnitude of the ionospheric conductivity leads to an unstable earthward convection and to its breaking into convection streams oriented at some angle to the initial convection direction. Because the instability can occur at the inner and outer edges of the plasma sheet, it may hold an explanation for formation of the famous double oval structure in auroral arc distribution (Akasofu, 1968; Elphinstone et al., 1995). The growth rate of this instability is maximum for structures with sizes less than the ion Larmor radius in the equatorial plane; in this case electrons can drift along such structures in a polarization electric field inside these structures, whereas ions do not participate in this motion.

This may lead to formation of relatively narrow auroral arcs with widths of the order of $10 \mathrm{~km}$. This instability is able to explain many features of such arcs including their conjugacy in opposite hemispheres. However, it cannot explain the very high growth rate of some auroral arcs and very narrow arcs (less than $1 \mathrm{~km}$ ) (Borovsky, 1993). For such arcs another type of instability must be considered.

\subsection{A model based on the modulation of precipitating electrons by field-aligned currents of the Alfven wave}

A self-consistent model for stratification of the magnetospheric convection in the lower magnetosphere first was proposed by Leontyev and Lyatsky (1982). This model is based on the idea that when the Alfven wave, emitted from a band of enhanced conductivity in the ionosphere, reaches an altitude of about $1 R_{E}$, where a particle acceleration region is thought to exist, fieldaligned currents of the wave may modulate particle acceleration.

In accordance with Leontyev and Lyatsky (1982), we assume the existence of a region of field-aligned electron acceleration at the altitude of about $1 R_{E}$ (e.g., Olsson et al., 1996). We also assume that field-aligned currents of the Alfven wave generated by an ionospheric inhomogeneity, coming into this acceleration region, produce an additional downward electron acceleration in the region of upward field-aligned current and their braking in the region of downward field-aligned current.

The growth of conductivity structures takes place when the ionization function associated with upward field aligned current is shifted from the edges of these structures toward their centers. Such a shift arises 


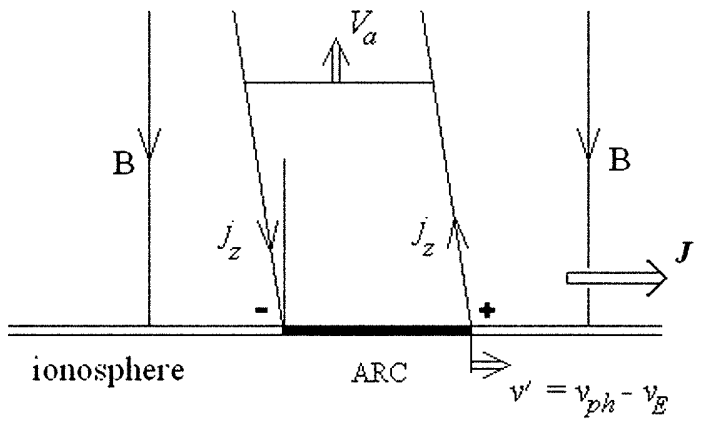

Fig. 7. Generation of an Alfven wave by a moving band of enhanced conductivity, which is assumed to be an auroral arc. The currents over the moving wave are inclined relative to the magnetic field $\mathbf{B}$ at some angle. $\mathbf{J}$ is the ionospheric current, and it is assumed that the band moves at a velocity $v^{\prime}=v_{p h}-v_{E}$ different from the $\mathbf{E} \times \mathbf{B}$ drift velocity $v_{E}$ where $v_{p h}$ is the phase velocity of the band

because the structures move at a velocity different from the $\mathbf{E} \times \mathbf{B}$ drift. As a result, field-aligned currents of upward propagating Alfven waves at some altitude appear shifted with respect to the edges of the structures as shown in Fig. 7. Leontyev and Lyatsky (1982) solved the problem numerically and showed that the instability indeed may take place.

The dispersion equation for this instability can be obtained as follows. We use the continuity equation for ionospheric currents Eq. (1). The magnitude of fieldaligned currents in an Alfven wave propagating in the magnetosphere can be derived as follows (Maltsev et al., 1977; Leontyev and Lyatsky, 1982):

$j_{z}=\operatorname{div}_{\perp} \mathbf{J}_{m}=-\Sigma_{w} \frac{\partial E^{\uparrow}}{\partial x}$

where $\mathbf{J}_{m}$ are transverse magnetospheric currents in the Alfven wave, closing field-aligned currents, $E^{\uparrow}$ is the transverse electric field of the upward moving Alfven wave, $\Sigma_{w}$ is the magnetospheric wave conductivity $\Sigma_{w}=c^{2} / 4 \pi v_{A}$, where $c$ is the light velocity and $v_{A}$ is the Alfven velocity.

The equation for the plasma density in the E region can be written in the following form:

$\frac{\partial n}{\partial t}+\frac{\partial}{\partial x}\left(n v_{x}\right)=q-\alpha n^{2}$

where $v_{x}$ is the $x$-component of the convection velocity; $q$ is the ionization function, and $\alpha$ is the recombination factor. Assuming the ionization function to be proportional to the magnitude of upward field-aligned current $j_{z, A}$ at some point $A$ of the acceleration region just over the point $x$, where we derive the electron density (see Fig. 1), we can write

$q=\chi a j_{z, A}(t, x)=\chi a j_{z}\left(t-\delta t, x-v_{x} \delta t\right)$

where $j_{z}\left(t-\delta t, x-v_{x} \delta t\right)$ is the field-aligned current over the ionospheric level at time $t-\delta t$ and coordinate $x-v_{x} \delta t$. This evaluation allows us to take into account a time delay $\delta t$ and a spatial shift $v_{x} \delta t$ required for the wave generated in the ionosphere to reach the acceleration region at time $t$ at point $A$, as seen in Fig. 8a. The time delay $\delta t$ is equal to $z_{1} / v_{A}$, where $z_{1} \approx 1 R_{E}$ is
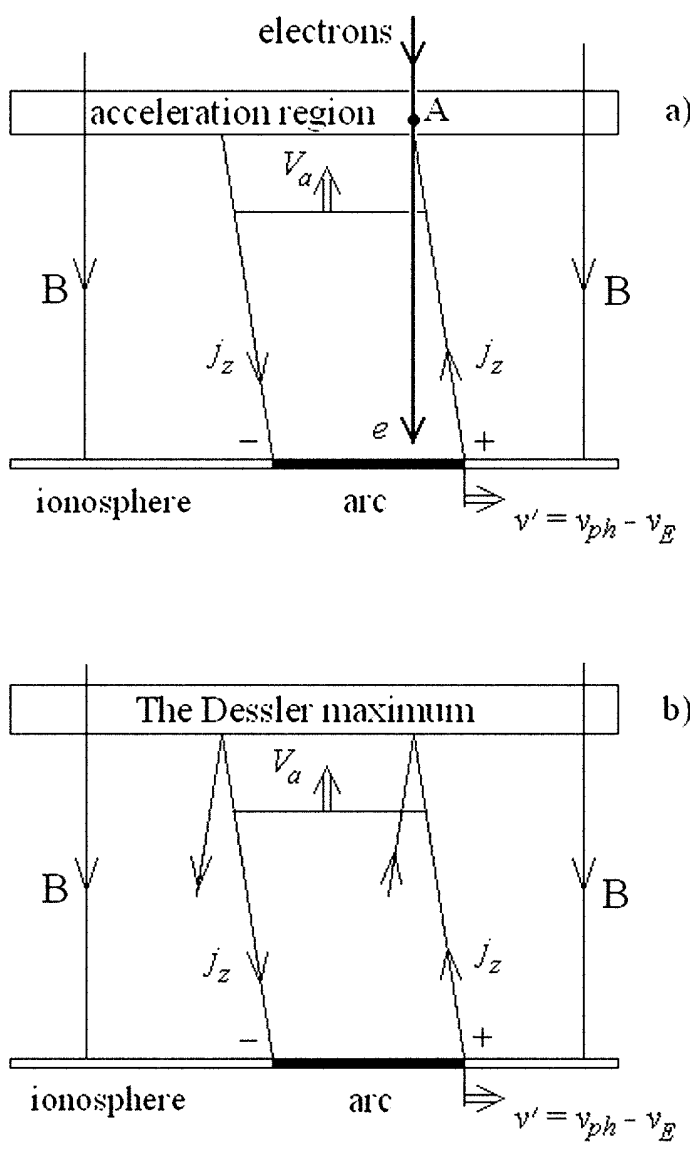

Fig. 8a, b. A scheme explaining the growth of a band of enhanced ionospheric conductivity, which is thought to be an auroral arc, for two models of magnetosphere-ionosphere coupling feedback instability in the lower magnetosphere. $j_{z}$ are field-aligned currents of the Alfven wave generated by the band. The first model $\mathbf{a}$ is based on the modulation of precipitating electrons by field-aligned currents of the upward moving Alfven wave (accelerated electrons are shown by symbol "e"). The second model b takes into consideration the reflection of the Alfven wave from the maximum of the Alfven velocity at about $3000 \mathrm{~km}$ altitude (the Dessler maximum). Growth of the structures takes place when the ionization function is shifted from the edges of enhanced conductivity structures toward their centers. Such shift arises when the structures move at a velocity $v^{\prime}=v_{p h}-v_{E}$ different from the $\mathbf{E} \times \mathbf{B}$ drift velocity $v_{E}$

the altitude of the acceleration layer. The factors $\chi$ and $a=1 / e \Delta z$ in Eq. (14) were defined earlier.

In the linear approximation for a plane wave $\exp [-i \omega t+i(\mathbf{k} \cdot \mathbf{r})]$, assuming $\Sigma_{P}^{\prime} / \Sigma_{P 0}=n^{\prime} / n_{0}$, where superscript " " " and subscript " 0 " mean perturbations and undisturbed values, respectively, we obtain from Eqs. (1), and (12)-(14) the following dispersion equation

$\omega-\mathbf{k} \cdot \boldsymbol{v}+i 2 \alpha n=\mathbf{k} \cdot \mathbf{A} e^{i(\omega-\mathbf{k} \cdot v) \delta t}$

where

$\mathbf{A}=\chi \frac{a}{n} \frac{\mathbf{J}_{e f}}{1+\Sigma_{P} / \Sigma_{w}}$

where $\mathbf{J}_{e f}=\Sigma_{P}\left[\mathbf{E}+\left(\Sigma_{H}^{\prime} / \Sigma_{P}^{\prime}\right) \mathbf{b} \times \mathbf{E}\right]$. When the Hall to Pedersen conductivity ratio $\Sigma_{H}^{\prime} / \Sigma_{P}^{\prime} \approx \Sigma_{H} / \Sigma_{P}$, the effective current $\mathbf{J}_{e f}$ is equal to the ionospheric current. 
The expression (15) can be separated into real and imaginary parts to give:

$\omega^{\prime}=\mathbf{k} \cdot \mathbf{A} \cos \omega^{\prime} \delta t$

$\gamma+2 \alpha n=\mathbf{k} \cdot \mathbf{A} \sin \omega^{\prime} \delta t$

where $\omega^{\prime}=\operatorname{Re}(\omega)-\mathbf{k} \cdot \boldsymbol{v}=k\left(v_{p h}-v_{x}\right)$. From Eq

for $\mathbf{k} \cdot \mathbf{A}>0$, the growth rate $\gamma$ is maximum when

$\omega^{\prime} \delta t \approx(\pi / 2-\varepsilon+2 m \pi)>0$

$\omega^{\prime} \delta t \approx-(3 \pi / 2-\varepsilon+2 m \pi)<0$

where $\varepsilon$ is a small value $\varepsilon \ll 1$, and $m=1,2,3, \ldots$ The upper expression is related to $\omega^{\prime}>0$ when $v_{p h}-v_{x}>0$; the lower expression is related to $\omega^{\prime}<0$ when $v_{p h}-v_{x}<0$. For negative $\mathbf{k} \cdot \mathbf{A}<0$ we have the opposite situation.

For the case of maximum growth rate, $\cos \omega^{\prime} \delta t \approx \varepsilon$ and $\sin \omega^{\prime} \delta t \approx 1$, and for the most important case $\omega^{\prime} \delta t \approx(\pi / 2-\varepsilon+2 m \pi)>0$ we obtain

$\gamma+2 \alpha n \approx \mathbf{k} \cdot \mathbf{A}$

The magnitude of $\mathbf{k}$ cannot be larger than $k_{\max }$ that corresponds to a minimum size of the structures, which is larger than several Larmor radii of accelerated electrons, which is of the order of few hundreds of meters.

For typical magnitudes $\chi=30, \Delta z=20 \mathrm{~km}$, $n=5 \cdot 10^{11} \mathrm{~m}^{-3}$, and $\mathbf{J}_{e f}=0.1 \mathrm{~A} / \mathrm{m}$ and $\Sigma_{P} / \Sigma_{w}=5$ (e.g., Kozlovsky and Lyatsky, 1999; Lyatsky et al., 1999), we obtain $A \approx 0.3 \mathrm{~km} / \mathrm{s}$. The most probable reason is the excitation of waves propagating along the ionospheric current. Because the growth rate is proportional to $\mathbf{k}$, this instability leads to the excitation of very narrow structures with sizes as small as $1 \mathrm{~km}$ and less. The phase velocity of these structures is close to the drift velocity of the plasma. The magnitude of the growth rate can be estimated by substituting in Eq. (20) the typical value of $A=0.3 \mathrm{~km} / \mathrm{s}$ as obtained earlier. We then obtain $\gamma \approx 300 \mathbf{k}$. Assuming $\mathbf{k}=10^{-3} \mathrm{~m}^{-1}$ yields $\gamma \approx 0.3 \mathrm{~s}^{-1}$.

We note that the motion of periodic structures at the $\mathbf{E} \times \mathbf{B}$ drift must lead to observations of magnetic pulsations. Assuming the wave length to be about $6 \mathrm{~km}$ and the drift velocity about $1 \mathrm{~km} / \mathrm{s}$ yields the pulsation period of about $6 \mathrm{~s}$. This is close to the expected period of the Alfven resonant oscillations in the lower magnetosphere. However, these oscillations are not Alfven resonant oscillations.

\subsection{A model based on the reflection of the Alfven wave (the Alfven resonant oscillation model)}

The model studying the instability in the global magnetosphere-ionosphere system was proposed by Sato and Holzer (1973), Sato (1978), Watanabe and Sato (1988) and others. Figure 8b explains the cause of the instability. A spatial shift of secondary upward fieldaligned currents in this model is provided by the reflection of Alfven waves from the opposite ionosphere. The motion of structures at a velocity $v^{\prime}$ relative to magnetospheric plasma leads to a displacement of fieldaligned currents of the reflected Alfven wave with respect to the original currents by a distance $\delta x=v^{\prime} 2 s / V_{A}$, where $s$ is the length of a magnetic field line, and $V_{A}$ is the Alfven velocity in the magnetosphere.

This model is based on a simple physical mechanism and has a great teaching impact; this was the first selfconsistent model for self-excitation of auroral arcs. Some of shortcomings of the model are the assumption that the Alfven wave propagates between opposite ionospheres without a strong damping, its failure to explain the existence of narrow auroral arcs, as well as its prediction of relatively low growth rate magnitudes.

Trakhtengertz and Feldstein (1984) and Lysak (1991) applied this model to examine the excitation of Alfven resonant oscillations between the ionosphere and a region of the enhanced Alfven velocity at an altitude of about $3000 \mathrm{~km}$, which is often called the Dessler maximum. The physics is absolutely the same as in the Sato and Holzer (1973) model, but the instability is believed to take place in the low magnetosphere.

The scheme explaining this instability is shown in Fig. 8b. The necessary shift of resulting field-aligned currents from the edges of the structures is provided by field-aligned currents of reflected Alfven waves shifted along the ionosphere due to the motion of the structures.

The dispersion equation for this instability can be obtained using the same method used for the previous case, but some equations must be exchanged. The equation for field-aligned currents of the reflected Alfven wave must be added, which is the same as Eq. (12) but with the opposite sign, so that instead of Eq. (12) we have

$j_{z}=j_{z}^{\uparrow}+j_{z}^{\downarrow}=\operatorname{div}_{\perp} \mathbf{J}_{m}=-\Sigma_{w} \frac{\partial E_{x}^{\uparrow}}{\partial x}+\Sigma_{w} \frac{\partial E_{x}^{\downarrow}}{\partial x}$

$E_{x}^{\downarrow}(t, x)=R E_{x}^{\uparrow}(t-\delta t, x-\delta x)$

where $j_{z}^{\uparrow}, j_{z}^{\downarrow}, E_{x}^{\uparrow}$, and $E_{x}^{\downarrow}$ are field-aligned currents and the electric field for upward $\left(^{\uparrow}\right.$ ) and downward $(\downarrow)$ waves, respectively. The total disturbed electric field is now the sum of both upward and downward waves $\mathbf{E}^{\prime}=$ $\mathbf{E}^{\uparrow}+\mathbf{E}^{\downarrow}$. The equation for ionization balance is the same as that of the previous case. However, the ionization function $q$ in this equation must now be written in some other form with the time delay being twice as long as that of the previous case (Fig. 8b).

The time delay $\delta t$ in Eq. (22) is equal now to $2 z_{2} / v_{A}$ where $z_{2}$ is the altitude of the Dessler maximum. For simplicity, we assume that the wave is reflected from a narrow layer at the altitude of about $3000 \mathrm{~km}$. This simplified approach allows us to obtain analytical expressions, which allows us to understand better the nature of the instability and to compare this model with those considered earlier. We note that this approach leads to an enhanced magnitude for the growth rate.

One more important feature of this model is that the factor $\chi$, the efficiency of ionization produced by one precipitating electron, is now equal to 1 . This model cannot consider accelerated particles because the 
acceleration takes place far from the ionosphere, and accelerated electrons do not follow the reflected Alfven wave. Taking into account a modification of electron acceleration by Alfven waves leads us immediately to the previous model considered. Thus, variations in the ionospheric conductivity in this model are produced not by accelerated electrons but by cold particles carrying field-aligned currents of the Alfven waves.

In the linear approximation for a plane wave $\exp [-i \omega t+i(\mathbf{k} \cdot \mathbf{r})]$, from Eqs. (1)-(6) and (21)-(22) we obtain the following dispersion equation

$\omega-\mathbf{k} \cdot v+i 2 \alpha n=-\frac{a}{n} \frac{\mathbf{k} \cdot \mathbf{J}_{e f}}{1+\frac{\Sigma_{p}}{\Sigma_{w}} \frac{1+\operatorname{Re}^{i \phi}}{1-\mathrm{Re}^{i \phi}}}$

where $R$ is the reflection coefficient for the Alfven wave and $\phi=\omega^{\prime} \delta t$, where again $\omega^{\prime}=\operatorname{Re}(\omega)-\mathbf{k} \cdot v$. The expression Eq. (18) can be rewritten for the real and imaginary parts of $\omega$ as follows:

$\operatorname{Re}(\omega)=\mathbf{k} \cdot v+\mathbf{k} \cdot \mathbf{A}_{1}\left(1+\frac{\Sigma_{P}}{\Sigma_{w}} \frac{1-R^{2}}{\left|1-\operatorname{Re}^{i \phi}\right|^{2}}\right)$

$\operatorname{Im}(\omega)=-\mathbf{k} \cdot \mathbf{A}_{1} \frac{\Sigma_{P}}{\Sigma_{w}} \frac{2 R \sin \phi}{\left|1-\operatorname{Re}^{i \phi}\right|^{2}}-2 \alpha n$

where

$\mathbf{A}_{1}=-\frac{a}{n} \frac{\mathbf{J}_{e f}}{\left|1+\frac{\Sigma_{P}}{\Sigma_{w}} \frac{1+\mathrm{Re}^{i \phi}}{1-\mathrm{Re}^{i \phi}}\right|^{2}}$

The magnitude of the growth rate is maximum for $\phi=\omega^{\prime} \delta t \approx(\pi / 2+2 m \pi)$, where $m=1,2,3, \ldots$. In this case we obtain

$\operatorname{Im} \omega=-\mathbf{k} \cdot \mathbf{A}_{1} \frac{2\left(\Sigma_{P} / \Sigma_{w}\right) R}{1+R^{2}}-2 \alpha n$

$\mathbf{A}_{1}=-\frac{a}{n} \frac{\mathbf{J}_{e f}}{1+\left(\frac{\Sigma_{P}}{\Sigma_{w}}\right)^{2}+2 \frac{\Sigma_{P}\left(1-R^{2}\right)}{\Sigma_{w}\left(1+R^{2}\right)}}$

The maximum value of the growth rate is related to $R=1$. In this case we obtain

$\operatorname{Im}(\omega)=-\frac{a}{n} \mathbf{k} \cdot \mathbf{J}_{e f} \frac{\left(\Sigma_{P} / \Sigma_{w}\right)}{1+\left(\Sigma_{P} / \Sigma_{w}\right)^{2}}-2 \alpha n$

For a typical magnitude of the ratio $\Sigma_{P} / \Sigma_{w} \approx 5$, the maximum magnitude of the growth rate appears to be $\chi$ times less than that given by expression Eq. (14) for the previous model. For more realistic conditions when the reflection coefficient is less than 1 , the growth rate becomes even smaller. Since the $\chi$ factor is about 30 , the Alfven resonator excitation model is also 30 times less effective than the model taking into consideration the modulation of electron acceleration by field-aligned currents of Alfven waves, considered.

Thus, in the last two models considered the instability arises because of generation of Alfven waves from arclike structures in the ionospheric conductivity. One model is based on the modulation of precipitating electrons by field-aligned currents of upward moving
Alfven waves. The other model takes into consideration the reflection of the Alfven wave from a maximum in the Alfven velocity at about $3000 \mathrm{~km}$ altitude. The growth of structures in both models takes place when the ionization function associated with upward field aligned current is shifted from the edges of enhanced conductivity structures toward their centers.

Although in principle both models may work, the growth rate for the first model based on the modulation of precipitating accelerated electrons, for typical conditions, is significantly larger than that of the second model based on the Alfven wave reflection. The mechanism based on the MIC instability in the lower magnetosphere is mostly suitable for the generation of narrow auroral arcs with widths of the order of $1 \mathrm{~km}$ and less. The growth rate of the instability for such structures can be as large as $1 \mathrm{~s}^{-1}$.

We note, that this mechanism enables us to explain auroral arc generation only in one ionosphere, and it is hardly suitable for generation of wide and conjugate auroral arcs; such arcs are likely excited in the global magnetosphere-ionosphere system as considered in Sect. 2.1. The proposed mechanism may be responsible for the formation of small-scale structures inside a wider arc. In this case, an original wide arc provides some original electron acceleration, which leads to favorable conditions for the instability in the lower magnetosphere.

We note that this instability may be responsible for the generation of both single and periodic structures. Figure 9 shows schematically these two possibilities. Figure $9 \mathrm{a}$ is related to the situation when the reflected Alfven wave comes back to the source band; in this case, single auroral arcs may be generated. Figure $9 \mathrm{~b}$ shows a possible scenario explaining the generation of periodic structures.

The phase velocity of the structures is close to the $\mathbf{E} \times \mathbf{B}$ drift. In the case of periodic structures, their motion must lead to the generation of magnetic pulsations with periods about $1-6 \mathrm{~s}$, which is close to the expected period of Alfven resonant oscillations in the lower magnetosphere. However, these oscillations (for the first model) are not real Alfven resonant oscillations.

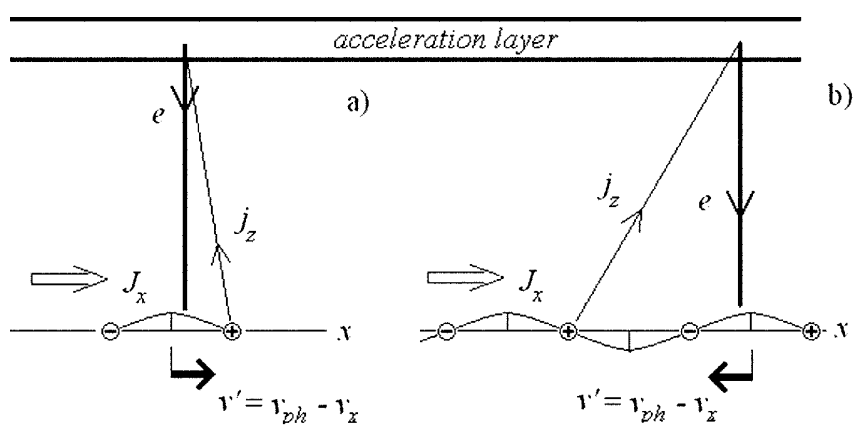

Fig. 9a, b. A scheme explaining the generation of a single arc-like structure and $\mathbf{b}$ periodic structures; $v^{\prime}=v_{p h}-v_{x}$ is the relative velocity of the structures in the coordinate system moving at the $\mathbf{E} \times \mathbf{B}$ drift velocity 


\section{Discussion and conclusions}

We have examined three models for the magnetosphereionosphere coupling (MIC) feedback instability. The first model is based on demagnetization of hot ions in the plasma sheet and the generation of magnetospheric currents, closing field-aligned currents in the vicinity of the inner and outer edges of the plasma sheet. This instability takes place in the global magnetosphereionosphere system. Two other models are based on modulation of particle precipitation by field-aligned currents of an Alfven wave generated by an ionospheric inhomogeneity, and on the reflection of this wave from the maximum in the Alfven velocity distribution at about $3000 \mathrm{~km}$ altitude, respectively. These two instabilities may take place in the lower magnetosphere, though the last model may be related to the global system as well.

We obtained the dispersion equations for all these three models for the magnetosphere-ionosphere coupling (MIC) feedback instability related to auroral arc generation, which allow us to investigate features of these instabilities and compare their efficiencies. For the total MIC system including the magnetosphere and both ionospheres, we considered a model relevant to the inner edge of the plasma sheet, which is different from that considered by Kozlovsky and Lyatsky (1999) for the far plasma sheet, and the obtained dispersion equation is different from that obtained by Kozlovsky and Lyatsky (1999). We obtained also dispersion equations for the two lower-magnetosphere instabilities, which allowed us to compare their efficiencies. We note that these instabilities were earlier examined by simulations (e.g., Leontyev and Lyatsky, 1982; Lysak, 1991) or in very complicated model (Trachtengertz and Feldstein, 1984) hindering their comparison. Main results of the present work can be summarized as follows.

1. The ion demagnetization model takes place when magnetospheric electrons drift through a spatial gradient of a hot magnetospheric ion population. This drift leads to the formation of field-aligned currents. The effect of upward field-aligned currents on particle precipitation and on the magnitude of the ionospheric conductivity leads to an instability of the earthward convection and to its breaking into convection streams oriented at some angle to the initial convection direction.

a. This instability develops in the global magnetosphere-ionosphere system though it may also take place in one hemisphere only. Because the source of this instability is the convection flow in the plasma sheet, it can be associated with a large energy release. The Poynting vector for this instability is downward (toward the Earth).

b. The growth rate of this instability is maximum for small-scale structures with sizes less than the ion Larmor radius in the equatorial plane. In this case, a polarization electric field across these structures leads to an electron $\mathbf{E} \times \mathbf{B}$ drift along these structures, whereas ions do not participate in this motion because of their large
Larmor radius. The characteristic time for instability development is restricted by the Alfven wave propagation time that is about $1 \mathrm{~min}$.

c. This model predicts that field-aligned currents over the arc must coincide with the electron precipitation flow.

d. A favorable situation for the instability takes place at the inner and outer boundaries of the plasma sheet where relatively cold magnetospheric electrons move earthward through a radial gradient of hot ions. This may explain the formation of the famous double oval structure in the auroral distribution.

e. This model may lead to formation of auroral arcs with widths of the order of $10 \mathrm{~km}$. It allows us to explain many features of such arcs including their good conjugacy in opposite hemispheres. However, this instability cannot explain the very high growth rate of some auroral arcs as well as the existence of very narrow arcs (less than $1 \mathrm{~km}$ ). For such arcs another type of instability must be examined.

2. The second model, leading to the stratification of convection flow is based on the modulation of particle precipitation by field-aligned currents of an Alfven wave generated by an ionospheric inhomogeneity. It may take place in the lower magnetosphere between the ionosphere and a region of electron acceleration at about $1 R_{E}$. Because of the motion of ionospheric structures at velocities different from the $\mathbf{E} \times \mathbf{B}$ plasma drift, the precipitating electron flux and the ionization function may be shifted by some distance with respect to the initial field-aligned currents. This produces the growth of conductivity structures.

a. This instability takes place in the lower magnetosphere. The source of this instability is located in the ionosphere, and the Poynting vector is expected to be upward (away from the Earth). We note that, although the original energy source is located in the magnetosphere (it can be the magnetospheric convection), however, any inhomogeneity of the ionospheric conductivity becomes a secondary energy source because energy, reaching this region from the magnetosphere, will propagate upward along the magnetic field as the Alfven wave.

b. The growth rate of the instability is maximum for small-scale structures with sizes less than the accelerated electron Larmor radius. The magnitude of the characteristic time for instability development may reach the time needed for the Alfven wave propagation from the ionosphere to the acceleration region, which is of the order of few seconds.

c. Field-aligned currents over the arc must be shifted toward the center of the arc with respect to the electron precipitation flow.

d. This model leads to the formation of very narrow auroral arcs with widths of the order of $1 \mathrm{~km}$ and less, and it can be responsible for the development of a fine structure inside a wide arc.

e. Motion of the periodic structures at the $\mathbf{E} \times \mathbf{B}$ drift velocity must lead to the generation of magnetic pulsations with periods of about $6 \mathrm{~s}$, which is close to the expected period of the Alfven resonant oscillations in the lower magnetosphere. However, these oscillations 
are not Alfven resonant oscillations. Therefore, observations of similar oscillations (e.g., Boesinger et al., 1999) cannot necessarily be interpreted as Alfven resonant oscillations.

3. The third model is based on the reflection of Alfven waves emitted by an ionospheric inhomogeneity from the opposite ionosphere or from the maximum in the Alfven velocity distribution at about $3000 \mathrm{~km}$ altitude (the Dessler maximum). This instability may take place both in the global magnetosphere and in the lower magnetosphere, where it can be responsible for generation of inhomogeneities connected with Alfven resonance oscillations between the ionosphere and the Dessler maximum in the Alfven velocity. In both cases, the efficiency of this mechanism is less than that for the models considered above. For example, for the case of the instability in the lower magnetosphere, the Alfven resonant excitation model is about 30 times less effective than the model taking into consideration the modulation of electron flux by field-aligned currents of Alfven waves, considered already. This is because the first model is suitable only for cold precipitating electrons, which have small ionization efficiency, whereas the second model may be associated with the modulation of accelerated electrons. The Alfven resonant oscillation model cannot be associated with accelerated electrons because they are accelerated far above the ionosphere and do not follow the field-aligned currents of the Alfven wave as taken at the ionospheric level. Nevertheless, for some situations this mechanism may also work.

In conclusion, we would like to say that the magnetosphere-ionosphere coupling mechanism considered in the present work is possibly not only possible mechanism for auroral arc generation, and some faint, subvisual arcs are possibly generated by another mechanism, without the effect of ionospheric conductivity, although this point requires further study.

Acknowledgements. Funding for this study was providing by the Canadian Natural Science and Engineering Research Council (NSERC). The work of W.L. was supported also by the Russian Foundation for Basic Research (grant 97-05-65894).

Topical Editor M. Lester thanks P. Janhunen and M. P. Freeman for their help in evaluating this paper.

\section{References}

Akasofu, S.-I., Polar and magnetospheric substorms, D. Reidel, Norwell, Mass., 1968

Boesinger, T., P. P. Belyaev, and M. T. Rietveld, Indications of ionospheric Alfven resonator effects in spectra of ionospheric heating induced magnetic disturbances, Books of abstracts of the 9th Int. EISCAT Workshop, Wernigerode, Germany, 6-10 Sept., 1999, Max-Planck Institut fur Aeronomie, 1999, p.11.

Borovsky, J. E., Auroral arc thickness as predicted by various theories, J. Geophys. Res., 98, 6101-6138, 1993.

Elphinstone R. D. et al., The double oval UV auroral distribution: 2. The most poleward arc system and the dynamics of the magnetotail, J. Geophys. Res., 100, 12 093-12 102, 1995.
Jaggi, R. K., and R. A. Wolf, Self-consistent calculation of the motion of a sheet of ions in the magnetosphere, J. Geophys. Res., 78, 2852-2866, 1973.

Kozlovsky, A. E., and W. B. Lyatsky, Instability of the magnetosphere-ionosphere convection and formation of auroral arcs, Ann. Geophysicae, 12, 636-641, 1994.

Kozlovsky, A., and W. Lyatsky, Finite Larmor radius convection instability in the near-Earth plasma sheet, J. Geophys. Res., 104, 2443-2449, 1999.

Leontyev, S. V., and W. B. Lyatsky, Solitary auroral arc generation, Planet. Space Sci., 30, 1-4, 1982.

Lyatsky, W. B., Conductivity waves in the magnetosphereionosphere system, Geomagn. Aeron., 27, 965-970, 1987.

Lyatsky, W., A possible role of ion demagnetization in substorm generation, J. Geophys. Res., 104, 19 905-19 910, 1999.

Lyatsky, W., and A. M. Hamza, The role of ion demagnetization in the plasma sheet in auroral arc and substorm generation, in 'Challenge to long-standing unsolved space physics problems in the 20th Century', Eds. K. De Jager, and S.-I. Akasofu, Space Sci. Rev., accepted 2000.

Lyatsky, W. B., and Y. P. Maltsev, Magnetosphere-Ionosphere Interaction, Moscow, Nauka, Russia, 1983.

Lyatsky, W., R. D. Elphinstone, Q. Pao, and L. L. Cogger, Field line resonance interference model for multiple auroral arc generation, J. Geophys. Res., 104, 263-268, 1999.

Lysak, R. L., Feedback instability of the ionospheric resonant cavity, J. Geophys. Res., 96, 1553-1568, 1991.

Maltsev, Y. P., W. B. Lyatsky, and A. M. Lyatskaya, Currents over the auroral arc, Planet. Space Sci., 25, 53-57, 1977.

Newell, P. T., C.-I. Meng, and K. M. Lyons, Superpression of discrete aurora by sunlight, Nature, 381, 766-767, 1996a.

Newell, P. T., K. M. Lyons, and C.-I. Meng, A large survey of electron acceleration events, J. Geophys. Res., 101, 2599-2614, 1996b.

Olsson, A., L. Andersson, A. I. Eriksson, J. Clemmons, R. E. Erlandson, G. Reeves, T. Hughes, and J. S. Murphree, Freja studies of the current-voltage relation in substorm-related events, J. Geophys. Res., 103, 4285-4301, 1998.

Potemra, T. A. Sources of large-scale currents, in Physical signatures of magnetospheric boundary layer processes, Eds. J. A. Holtet, and A. Egeland, Kluwer, Norwell, Mass., 1994.

Roux, A., S. Perraut, P. Robert, A. Morane, A. Pedersen, A. Korth, G. Kremser, B, Aparicio, D. Rodgers, and R. Pellinen, Plasmasheet instability related to the westward travelling surge, J. Geophys. Res., 96, 17 696-17 714, 1991.

Samson, J. C., A. K. MacAulay, R. Rankin, P. Fricz, I. Voronkov, and L. L. Cogger, Substorm intensifications and resistive flowballooning instabilities in the near-Earth magnetotail, in Proc. of the Third International Conference on Substorm (ICS-3), Eur. Space Agency Spec. Publ. ESA SP-389, 399-404, 1996a.

Sato, T., A theory of quiet auroral arcs, J. Geophys. Res., 83, 1042 1048, 1978.

Sato, T., and T. E. Holzer, Quiet auroral arcs and electrodynamic coupling between the ionosphere and the magnetosphere, J. Geophys. Res., 78, 7314-7329, 1973.

Trakhtengertz, V. Y., and A. Y. Feldstein, Quiet auroral arcs: Ionosphere effect of magnetospheric convection stratification, Planet. Space Sci., 32, 127-134, 1984.

Volkov, M. A., W. B. Lyatsky, and Y. P. Maltsev, On structure of electric field and currents in region of Harang discontinuity, Geomagn. Aeron., 25, 445-449, 1985.

Watanabe, K., and T. Sato, Self-excitation of auroral arcs in a three-dimensional coupled magnetosphere-ionosphere system, Geophys. Res. Lett. 15, 717-720, 1988.

Wolf, R.A., Magnetospheric configuration, in Introduction to Space Physics, Eds. M. G. Kivelson and C. T. Russell, Cambridge University Press, pp. 288-329, 1995. 